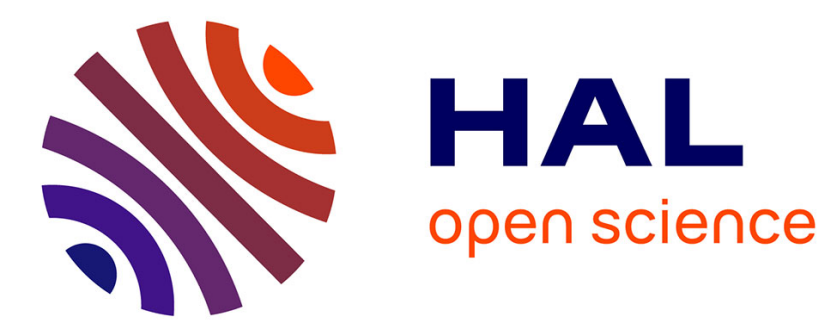

\title{
Identification of a managed river reach by a Bayesian approach
}

Magalie Thomassin, Thierry Bastogne, Alain Richard

\section{To cite this version:}

Magalie Thomassin, Thierry Bastogne, Alain Richard. Identification of a managed river reach by a Bayesian approach. IEEE Transactions on Control Systems Technology, 2009, 17 (2), pp.353-365. 10.1109/TCST.2008.2000982 . hal-00341280

\section{HAL Id: hal-00341280 \\ https://hal.science/hal-00341280}

Submitted on 24 Nov 2008

HAL is a multi-disciplinary open access archive for the deposit and dissemination of scientific research documents, whether they are published or not. The documents may come from teaching and research institutions in France or abroad, or from public or private research centers.
L'archive ouverte pluridisciplinaire HAL, est destinée au dépôt et à la diffusion de documents scientifiques de niveau recherche, publiés ou non, émanant des établissements d'enseignement et de recherche français ou étrangers, des laboratoires publics ou privés. 


\title{
Identification of a Managed River Reach by a Bayesian Approach
}

\author{
Magalie Thomassin, Thierry Bastogne, and Alain Richard, Member, IEEE
}

\begin{abstract}
This paper considers the problem of identification, and more particularly of time-delay estimation, of a river reach managed to produce hydroelectric power. Difficulties lie in the obligation to use data collected during a combined feedback/feedforward control carried out by a human operator. We propose a Bayesian identification method, nonsupervised and simple to implement, estimating jointly the time-delay and a finite impulse response (FIR). It is based on the detection of an abrupt change in the FIR at a time equal to the time-delay. Experimental results show the effectiveness of the proposed method to estimate the river reach time-delay from data collected in imposed experimental conditions.
\end{abstract}

Index Terms

Delay estimation, Bayesian identification, degeneracy, impulse response, open-channel system.

\section{INTRODUCTION}

The problem addressed in this paper is the identification of a managed river reach, described in Fig. 1, and more precisely the estimation of the time-delay between the inflow rate and the downstream water level of the reach. Several works dealing with modeling and identification of open water channels have been already published [1]-[8]. In [9]-[11], second and third order plus time-delay non linear models have been developed. However, few applications concern the time-delay estimation in this type of plant [12], although it directly influences the control performances and, in particular, the control stabilization [13]. This is particularly true for cascaded systems like run-of-river hydroelectric plants [14]. In practice, time-delays are either empirically estimated from knowledge of operators or experimentally determined from the measurement of intumescence propagation time. But in both cases, the estimates are still characterized by a large uncertainty.

For safety precautions and economic reasons, e.g. flood risks or fall-off in hydroelectric power production, the implementation of experimental protocols is not possible. In this case, estimation data are collected under normal

M. Thomassin is with the University of Bordeaux, laboratoire de l'Intégration du Matériau au Système (IMS), CNRS UMR 5218, 351 cours de la libération, 33405 Talence Cedex, France (e-mail: magalie.thomassin@laps.ims-bordeaux.fr).

T. Bastogne and A. Richard are with the Centre de Recherche en Automatique de Nancy (CRAN), Nancy-Université, CNRS, UMR 7039, Faculté des sciences et techniques, BP239, 54506 Vandœuvre-lès-Nancy Cedex, France (e-mail: thierry.bastogne@cran.uhp-nancy.fr; alain.richard@cran.uhp-nancy.fr). 
operating conditions, i.e. in a context of water level regulation carried out by a human operator. The purpose is, on the one hand, to estimate the nominal value of the time-delay and, on the other hand, to determine its evolution over one year. Indeed, the time-delay is a flow-variant parameter and the mean flow rate of the reach can be multiplied by ten during a year. The difficulty is twofold: 1) taking into account the actions of the human operator; and 2) compensating the lack of information in the estimation data sets. Indeed, if the operator actions are not considered (closed-loop system identification by a direct approach [15]), then most of time-delay estimation methods, like the exhaustive search based on the minimization of the output error, fail due to correlations between the input and the output data [16], [17]. Moreover, it is shown in this paper that human operators introduce a feedforward compensation to anticipate output variations. This feedforward control "hides" the reach time-delay in the transfer function of the closed-loop system. The first coefficients of its impulse response are not equal to zero. So, classical time-delay estimation methods, like the method using a shift operator model with an expanded numerator polynomial [18], are inappropriate.

The problem addressed herein is the identification and time-delay estimation of systems equipped with combined feedback/feedforward control systems (carried out by human operators) subject to large uncertainties (human operator is changed every 8 hours) and from few informative data sets, collected during regulation. To overcome these difficulties, a Bayesian method which jointly estimates the time-delay and a finite impulse response (FIR), is proposed. It is based on the fact that the time-delay introduces a discontinuity in the impulse response. In presence of few informative data, the FIR identification is an ill-conditioned inverse problem. The Bayesian approach provides a coherent and complete framework to take into account some prior knowledge. The latter corresponds classically in the case of a FIR estimation to a temporal smoothness constraint imposed by using a differentiation matrix [19]. In our problem, the FIR is slowly time-variant except at a time equal to the time-delay. The joint estimation of the FIR and the abrupt change instant are then performed by modifying the differentiation matrix. Unlike some

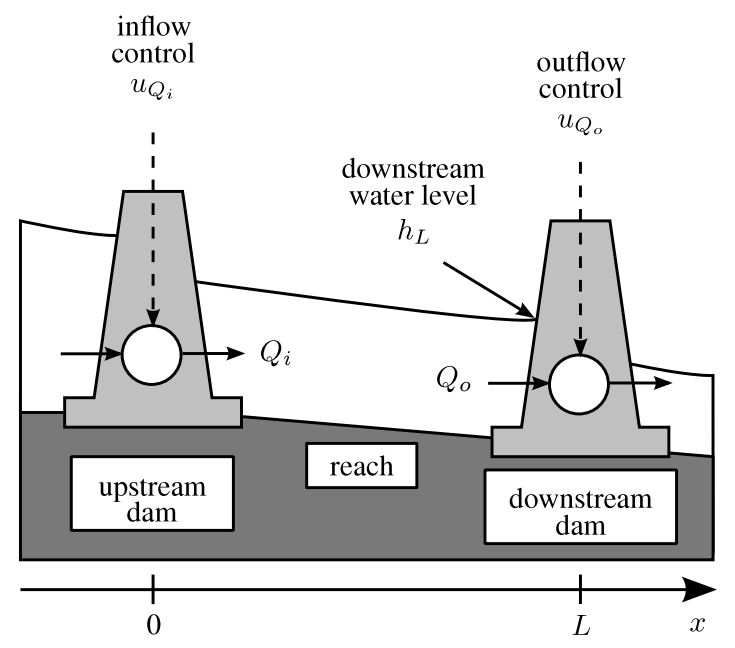

Fig. 1. River reach managed to produce hydroelectric power. 
classical approaches which first estimate a FIR and then detect a discontinuity, this method requires only one step. The choice of the FIR model structure allows to limit the problem of model uncertainties introduced by the human operator.

The paper is organized as follows. In section II, the variation range of the time-delay is evaluated from average hydraulic characteristics. A simplified modeling of a managed river reach is then presented in section III. The simplified model points out the main issues of the identification problem of this process. In section IV, a Bayesian method which jointly estimates the time-delay and the finite impulse response is proposed. During the hyperparameters estimation step, a degeneracy of a probability density function, leading to unreal results, is highlighted in section V and a solution is developed. In section VI, the identification method is applied to the data sets collected from a reach of the 'Basse-Isère' river, in France, over one year and a time-day representation of the estimated impulse responses is presented. This original description allows to clearly observe the evolution of the impulse response (and of the time-delay) over the year. Finally, the application results are analyzed and compared with empirical knowledge.

\section{A PRIORI VARIATION RANGE OF THE TIME-DELAY FROM HYDRAULIC ANALYSIS}

The propagation of shallow water waves in channel with rectangular cross section is controlled by the various forces included in the equation of motion

$$
\frac{1}{g} \frac{\partial v}{\partial t}+\frac{v}{g} \frac{\partial v}{\partial x}+\frac{\partial z}{\partial x}+\left(S_{f}-S_{b}\right)=0
$$

where $v$ is the velocity averaged in a vertical section, $z$ is the depth of flow, $g$ is the acceleration of gravity, $S_{f}$ is the friction slope and $S_{b}$ is the bed slope. Ponce and Simons in [20] give the expression of the waves celerity

$$
c=\left(1+c_{r}\right) v^{*}
$$

where $v^{*}$ is the mean flow velocity and $c_{r}$, the relative celerity, verifies

$$
0.5 \leqslant c_{r} \leqslant 1 / F_{o} \quad \text { if } F_{o}<2
$$

where $F_{o}$ is the Froude number

$$
F_{o}=\frac{v^{*}}{\sqrt{g z^{*}}}
$$

with $z^{*}$ the mean depth. As a consequence, the propagation time of waves $\tau_{h}$, equal to $L / c$ where $L$ is the reach length, in a straight channel with rectangular cross section, verifies

$$
\frac{L}{\left(1+\frac{1}{F_{o}}\right) v^{*}} \leqslant \tau_{h} \leqslant \frac{L}{(1+0.5) v^{*}} \quad \text { if } F_{o}<2 .
$$

The lower bound corresponds to the propagation time of a gravity wave, whereas the upper bound corresponds to the one of a kinematic wave.

Let consider now the hydraulic characteristics of the reach. Table I gives its main physical specifications. Other values are not available. Fig. 2 gives the mean daily discharge over the year (in downstream). It lies from about 
TABLE I

CHARACTERISTIC VALUES OF THE REACH

\begin{tabular}{cc}
\hline length $L$ & $8.2 \mathrm{~km}$ \\
average width $l^{*}$ & $81.6 \mathrm{~m}$ \\
average depth $z^{*}$ & $4.9 \mathrm{~m}$ \\
surface area $A$ & $6.7 \times 10^{5} \mathrm{~m}^{2}$ \\
\hline
\end{tabular}

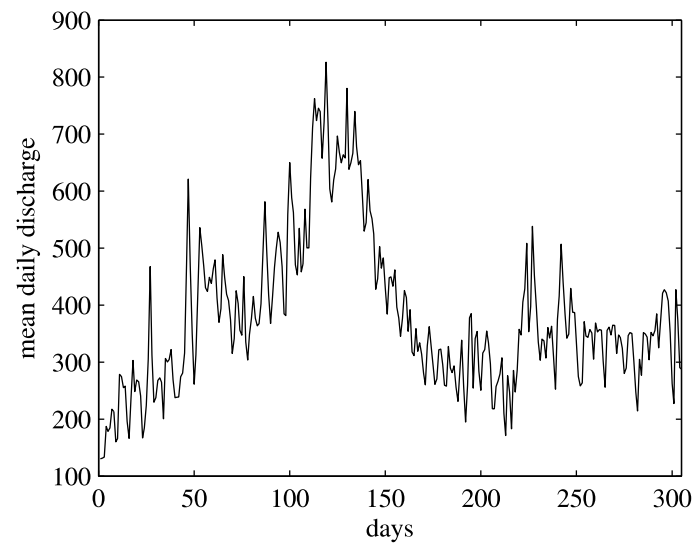

Fig. 2. Mean daily discharge (in $m^{3} / s$ ) over the year

$100 \mathrm{~m}^{3} / \mathrm{s}$ to $800 \mathrm{~m}^{3} / \mathrm{s}$, but most are lower than $500 \mathrm{~m}^{3} / \mathrm{s}$. The mean variation (peak-to-peak) over one day is about $150 \mathrm{~m}^{3} / \mathrm{s}$. Fig. 3 gives the evolution of the mean daily level (in downstream with reference to NGF-IGN69) over the year. The down peaks correspond to an increase of the downstream flow rate (downstream dam release). Over one day, the mean variation is about $0.2 \mathrm{~m}$. Fig. 4 represents the evolution of the mean daily Froude number

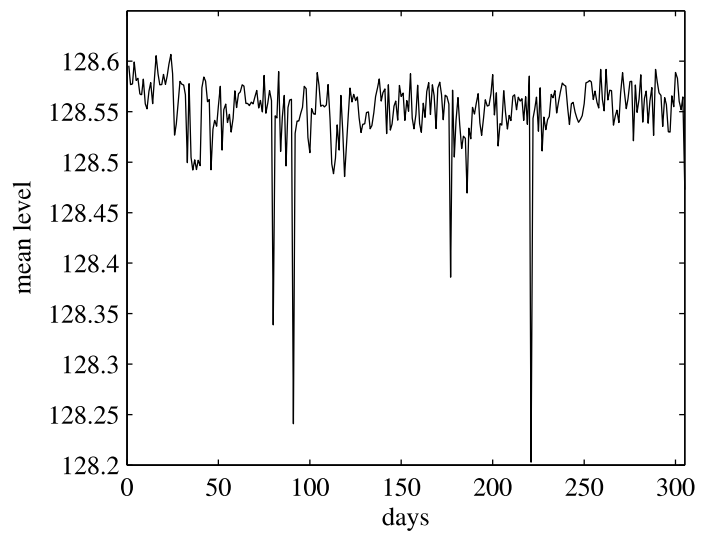

Fig. 3. Mean daily level (in $m$ with reference to NGF-IGN69) over the year 


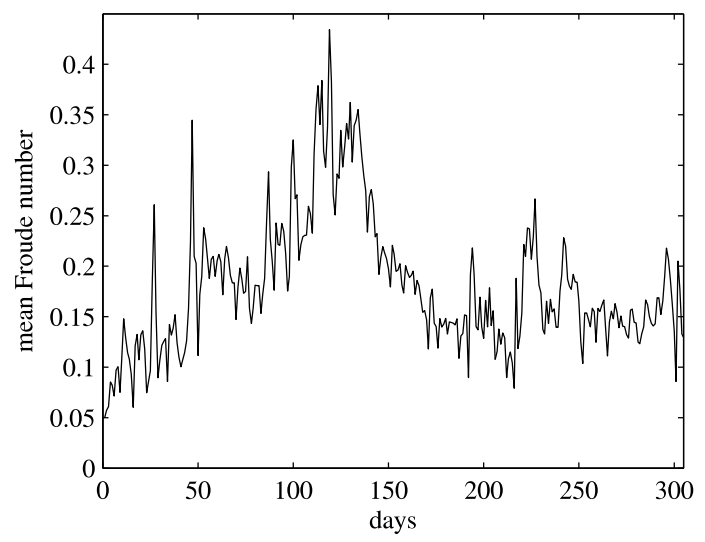

Fig. 4. Mean daily Froude number over the year

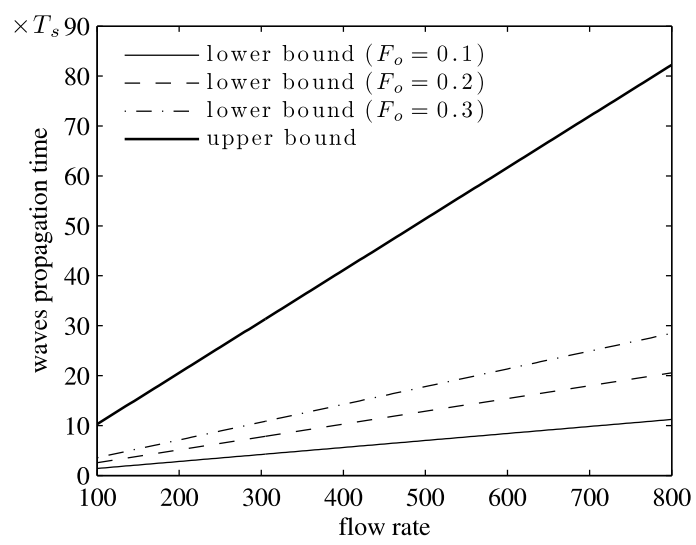

Fig. 5. Bounds of the waves propagation time $\left(T_{s}=133 \mathrm{~s}\right)$ versus the flow rate (in $\mathrm{m}^{3} / \mathrm{s}$ )

over the year. The Froude number can be interpreted as the ratio of the inertial to gravity forces in the flow. The mean daily Froude number is obtained by the relation

$$
F_{o}^{*}=\frac{v^{*}}{\sqrt{g z^{*}}}=\frac{Q^{*}}{S^{*} \sqrt{g z^{*}}}=\frac{Q^{*}}{l^{*} z^{*} \sqrt{g z^{*}}}
$$

where $v^{*}$ is the mean daily velocity, $Q^{*}$ is the mean daily discharge, $S^{*}=l^{*} z^{*}$ is the mean daily section area, $l^{*}$ is the average width of the reach section and $z^{*}$ is the mean daily level. The mean Froude number is lower than 2, therefore the propagation time verifies (5). Its bounds are plotted in Fig. 5 versus the mean flow rate in order to evaluate its variation. It can vary from few sampling periods (with $T_{s}=133 \mathrm{~s}$ ) for gravity waves to several tens for kinematic waves. Thus the propagation time of kinematic waves depends much more on the flow rate than the one of gravity waves (especially if $F_{o}$ is weak). Fig. 6 shows the daily lower and upper bounds of the propagation time evaluated from the daily data sets (or more precisely from mean daily flow rates - Fig. 2- and mean daily Froude numbers - Fig. 4-). Small variations (betwen $7 T_{s}$ and about $8.5 T_{s}$ ) of the lower bounds are observed for gravity waves. However, the upper bounds (for kinematic waves) range from $20 T_{s}$ to more $100 T_{s}$, which is 


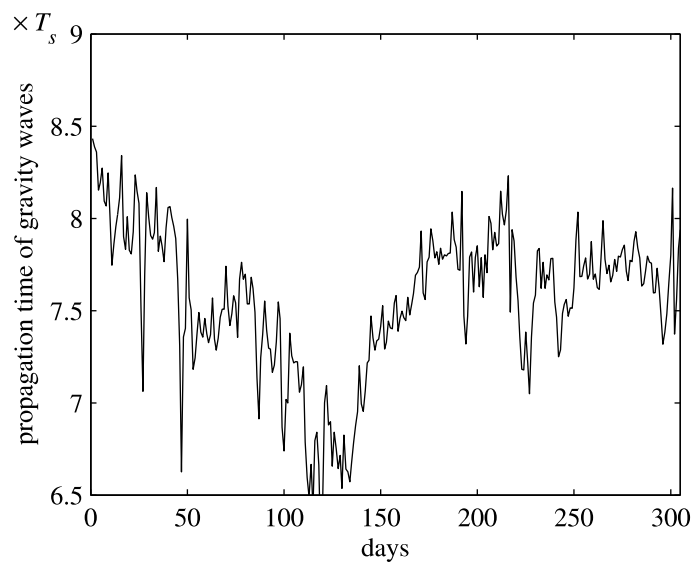

(a) lower bounds

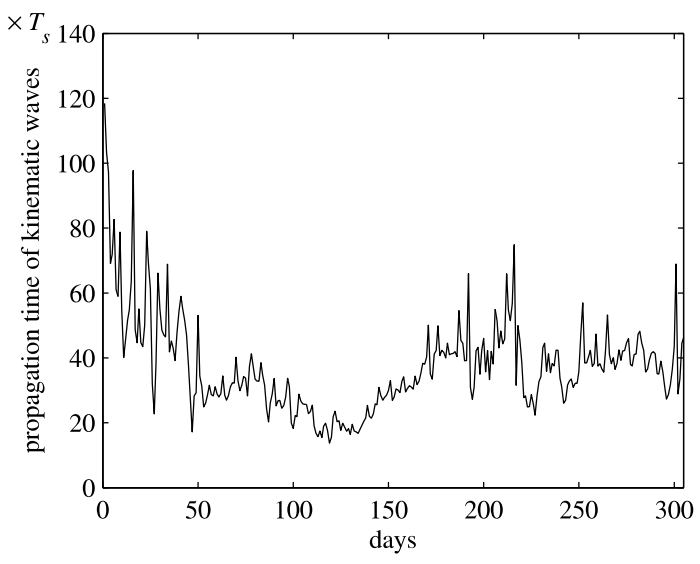

(b) upper bounds

Fig. 6. Bounds of the waves propagation time for each day $\left(T_{s}=133 \mathrm{~s}\right)$

not realistic in this case.

In conclusion, the fastest waves are the gravity waves. Their propagation time fluctuates between 7 and $8 T_{s}$. However, these results are obtained in an ideal case of a straight rectangular channel with average values whereas the geometry of the studied reach is very space-variant. For information, note that the reference value of the time-delay usually used by operators for the manual control of the reach is between $5 T_{s}$ and $11 T_{s}$.

\section{SIMPLIFIED MODELING OF THE RIVER REACH}

In this section, a simplified model of the river reach is developed to explicitly state the time-delay estimation problem in this practical framework and to explain failures of usual estimation approaches.

The river reach is described by a continuous-time model in which the input variables are the inflow and outflow rates, respectively denoted by $Q_{i}$ and $Q_{o}$, and the output variable is the downstream water level $h_{L}$. The inflow and outflow rates are not measured, but the control signals $u_{Q_{i}}$ and $u_{Q_{o}}$ are known. A one-day data set is presented in Fig. 7 and the main process variables are summarized in table II. 

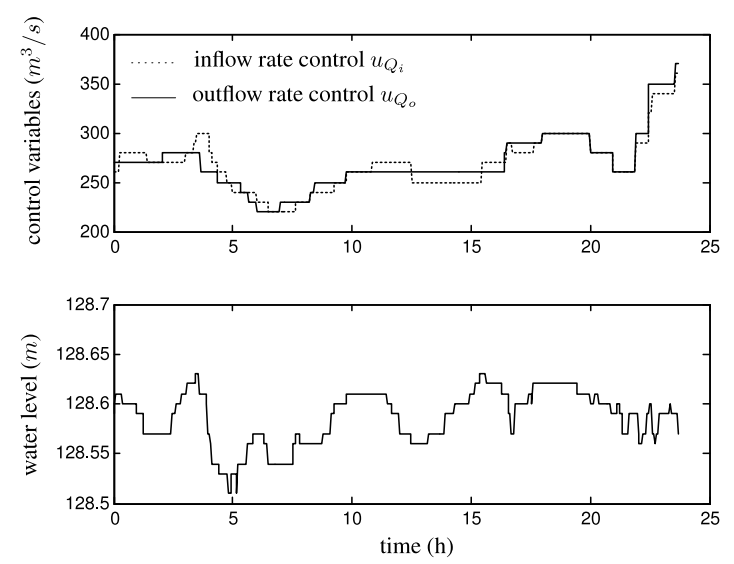

Fig. 7. A one-day estimation data set

TABLE II

MAIN PROCESS VARIABLES.

\begin{tabular}{cl}
\hline$u_{Q}$ & Flow rate control of a dam \\
$Q$ & Real flow rate of a dam \\
Subscript $i$ & Variable of the upstream dam (inflow) \\
Subscript $o$ & Variable of the downstream dam (outflow) \\
$h_{L}$ & Downstream water level \\
\hline
\end{tabular}

A river reach is an open-channel flow system. Its behavior can thus be modeled by the Saint-Venant equations (or shallow water equations) [21], [22]. However, these equations are difficult to apply in the case of a river mainly because the river geometry is seldom known and is generally space-variant [3] (see [7]-[11] for an application to an irrigation channel). Consequently, we have to use a simplified model allowing to reproduce the essential characteristics of the system at a given point of the reach (in our case at $x=L$, where $L$ is the reach length). This problem reduction allows us to transform a partial derivative equation into a differential one. Indeed, in the absence of tributary inflows, the river reach dynamics around an operating point can be approximately described by a model structure whose main elements are a time-delay and an integrator term [2]

$$
\overline{\Delta h_{L}}(s)=\frac{1}{A s}\left(e^{-\tau s} \overline{\Delta Q_{i}}(s)-\overline{\Delta Q_{o}}(s)\right)+\overline{\delta h_{L}}(s)
$$

with notations given in table III. $A$ is the reach water surface (in $m^{2}$ ) and $\tau$ denotes the unknown time-delay between the inflow rate and the downstream water level. The time-delay between the outflow rate and the downstream water level is fixed to zero because the water level measurement station is close to the downstream dam. This model describes the essential volume variation of the reach, so it represents only the low frequency characteristics of the reach dynamics. Nevertheless, it is sufficient for the control purposes where the bandwidth is limited. As already seen in section II, the flow propagation time is a function of the mean level and of the mean flow rate. However, 
TABLE III

MAIN NOTATIONS.

\begin{tabular}{cl}
\hline$s$ & Laplace transform variable \\
$\bar{x}(s)$ & Laplace transform of the signal $x(t)$ \\
$\delta x$ & Modeling errors and measurement uncertainties of $x$ \\
$\Delta x$ & $=x-x_{0}$ with $x_{0}$ initial condition and stationary point \\
$q^{-1}$ & Shift operator \\
$T_{s}$ & Sampling period $(\approx 133 s)$ \\
$\hat{x}$ & Estimate of $x$ \\
\hline
\end{tabular}

considering the level variations of only a few centimeters (see Fig. 7) and a weak variation of the flow rate over a day, $\tau$ is supposed to be a slowly time-varying parameter that can be considered as constant over a day (duration of a data set). In the same way, considering the geometrical characteristics of the reach $\left(A \approx 640000 \mathrm{~m}^{2}\right)$ and the weak variations of the level and of the flow rate, the integration gain can also be considered as a constant parameter over a day. Nevertheless, the mean daily flow rate $Q^{*}$ can be multiplied by ten over the year (see Fig. 2). The consequences of this variation on $\tau$ are not well-known. This is another objective herein, i.e. to estimate the variation interval of $\tau$ over a year.

Each dam is equipped with flow control loops whose response time is negligible as compared to the sampling period $T_{s} \approx 133 s$. Consequently, these loops may be modeled by constant gains (denoted by $K_{i}$ and $K_{o}$ for the upstream and downstream dams, respectively) and a zero-order hold since flow rates are continuous-time signals whereas control signals are discrete-time ones.

After discretization ${ }^{1}$ and under the assumption that the time-delay is a multiple of the sampling period $\tau=d T_{s}$, the system "reach+dams" can be described by the following equation

$$
\Delta h_{L}[k]=\alpha_{i} \frac{q^{-d-1}}{1-q^{-1}} \Delta u_{Q_{i}}[k]-\alpha_{o} \frac{q^{-1}}{1-q^{-1}} \Delta u_{Q_{o}}[k]+v[k]
$$

where $\alpha_{i}=K_{i} T_{s} / A, \alpha_{o}=K_{o} T_{s} / A$ and $d \geqslant 1$ is the time-delay between $h_{L}$ and $u_{Q_{i}}$. The signal $v[k]$ represents the measurement, modeling and quantification errors.

In a managed river reach, the water levels at some critical points have to be controlled. In our case, there is only one critical point (corresponding to the downstream water level $h_{L}$ ). The water level regulation is carried out by a human operator who assigns outflow rate control values. The objective of the operator is to maintain the water level at a given setpoint by taking into account the water level measurements and by anticipating variations of the inflow rate. So, the operator performs a combined feedback/feedforward control depicted by the block diagram in Fig. 8. Feedback and feedforward actions of the operator are described by two proportional gains, respectively denoted by

\footnotetext{
${ }^{1}$ The discrete-time variables are denoted by $x[k]$ and correspond to the time sampling with a constant sampling period $T_{s}$ of the continuoustime variable $x(t): x[k]=x\left(k T_{s}\right)$.
} 


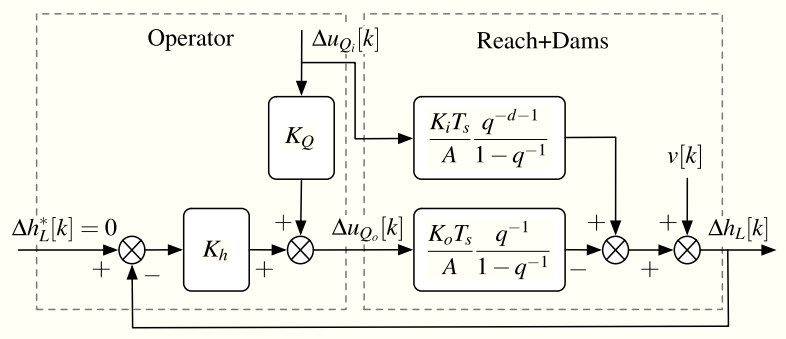

Fig. 8. Block-diagram of the managed river reach.

TABLE IV

ESTIMATION ALGORITHM OF $d$ WITH AN EXHAUSTIVE SEARCH.

$$
\begin{aligned}
& \diamond \text { For } d=d_{\min }, d_{\min }+1, \ldots, d_{\max }, \text { estimation of } \boldsymbol{\theta} \text { at } d \text { fixed: } \\
& \qquad \hat{\boldsymbol{\theta}}_{d}=\arg \min _{\boldsymbol{\theta}} J(\boldsymbol{\theta}, d) \\
& \diamond \text { Estimation of } d \text { at } \hat{\boldsymbol{\theta}}_{d} \text { fixed: } \hat{d}=\arg \min _{d \in \mathbb{D}\left(d_{\min }, d_{\max }\right)} J\left(\hat{\boldsymbol{\theta}}_{d}, d\right) .
\end{aligned}
$$

$K_{h}$ and $K_{Q}$

$$
\Delta u_{Q_{o}}[k]=K_{Q} \Delta u_{Q_{i}}[k]-K_{h} \Delta h_{L}[k]
$$

Theoretically, the feedforward action contains a time-delay which should counteract the process time-delay. However, in practice, it has been observed that operators usually tend to reject the disturbance effects by handling the control variable without waiting for its effect on the water level. Accordingly, the controller time-delay is fixed to zero. Note that (9) is a very simplified model of human operators who have not the same behavior and change every 8 hours.

Finally, a managed river reach, controlled by a human operator, is modeled by the block-diagram in Fig. 8 . The variation of the downstream water level reference $\Delta h_{L}^{*}[k]$ is assumed to be null (regulation mode). Accordingly, the model structure can be represented by the following expression

$$
\Delta h_{L}[k]=\underbrace{\frac{\alpha_{i} q^{-d}-K_{Q} \alpha_{o}}{-\left(K_{h} \alpha_{o}+1\right) q^{-1}+1} q^{-1}}_{H\left(q^{-1}\right)} \Delta u_{Q_{i}}[k]+\underbrace{\frac{1-q^{-1}}{-\left(K_{h} \alpha_{o}+1\right) q^{-1}+1} v[k]}_{w[k]} .
$$

The time-delay of the transfer function $H\left(q^{-1}\right)$ is equal to 1 and does not depend on $d\left(K_{Q} \alpha_{o} \neq 0\right)$.

A simple solution to estimate the time-delay $d$ is through an exhaustive search over $\mathbb{D}\left(d_{\min }, d_{\max }\right)=\left\{d \in \mathbb{N}^{*}\right.$ : $\left.d_{\min } \leqslant d \leqslant d_{\max }\right\}$, where $d_{\min }$ and $d_{\max }$ are known, as described by the algorithm in table IV. Two approaches can be applied in this context of feedback/feedforward model identification: 1) the direct approach which takes no account of the feedback/feedforward action and treats data $\Delta u_{Q_{i}}, \Delta u_{Q_{o}}$ and $\Delta h_{L}$ in the open loop context (8); and 2) the indirect approach which identifies the feedback/feedforward model (10) from the input $\Delta u_{Q_{i}}$ to the output $\Delta h_{L}$. Note that the direct approach has been successfully applied by [23] to identify an irrigation channel 


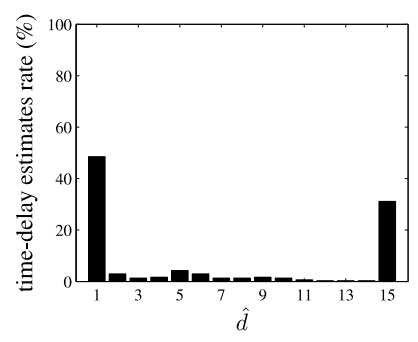

(a) direct approach

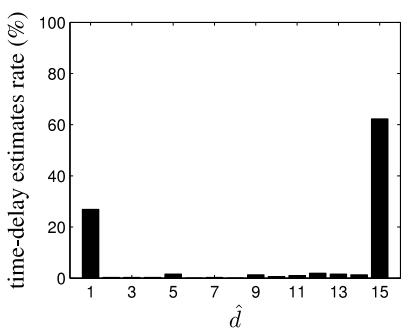

(b) indirect approach

Fig. 9. Histograms of the time-delay estimates.

(with a first order nonlinear model in which the time-delay is known) in a closed loop context (without feedforward action).

The criterion to minimize for the direct approach is the following

$$
\begin{aligned}
J(\boldsymbol{\theta}, d) & =\frac{1}{N} \sum_{k=0}^{N-1} \frac{1}{2}(\hat{v}[k, \boldsymbol{\theta}, d])^{2} \\
& =\frac{1}{N} \sum_{k=0}^{N-1} \frac{1}{2}\left(\Delta h_{L}[k]-\boldsymbol{\varphi}^{T}[k, d] \boldsymbol{\theta}\right)^{2}
\end{aligned}
$$

with $\boldsymbol{\theta}=\left[\alpha_{i}, \alpha_{o}\right]^{T}$ and $\varphi^{T}[k, d]=\left[\Delta u_{Q_{i}}^{f}[k-1-d],-\Delta u_{Q_{o}}^{f}[k-1]\right]$ where the superscript $f$ indicates that the signal is filtered by $\frac{1}{1-q^{-1}}: x^{f}[k]=\frac{1}{1-q^{-1}} x[k]$. This criterion has an explicit solution at a fixed $d$ (under invertibility condition)

$$
\widehat{\boldsymbol{\theta}}_{d}=\left[\sum_{k=0}^{N-1} \boldsymbol{\varphi}[k, d] \boldsymbol{\varphi}^{T}[k, d]\right]^{-1} \sum_{k=0}^{N-1} \boldsymbol{\varphi}[k, d] \Delta h_{L}[k] .
$$

For the indirect approach, the criterion to minimize is

$$
J(\boldsymbol{\theta}, d)=\frac{1}{N} \sum_{k=0}^{N-1} \frac{1}{2}\left(\Delta h_{L}^{f}[k]-\varphi^{T}[k, d] \boldsymbol{\theta}\right)^{2}
$$

with $\boldsymbol{\theta}=\left[K_{h} \alpha_{o}+1,-K_{Q} \alpha_{o}, \alpha_{i}\right]^{T}$ and $\varphi^{T}[k, d]=\left[\Delta h_{L}^{f}[k-1], \Delta u_{Q_{i}}^{f}[k-1], \Delta u_{Q_{i}}^{f}[k-d-1]\right]$. It has an explicit solution which is:

$$
\widehat{\boldsymbol{\theta}}_{d}=\left[\sum_{k=0}^{N-1} \boldsymbol{\varphi}[k, d] \boldsymbol{\varphi}^{T}[k, d]\right]^{-1} \sum_{k=0}^{N-1} \boldsymbol{\varphi}[k, d] \Delta h_{L}^{f}[k] .
$$

We have at our disposal 332 data sets, relative to as many days, i.e. $N \approx 650$ (see Fig. 7 for a data set example). Fig. 9a represents the histogram of the time-delay estimates for the direct approach with $d_{\min }=1$ and $d_{\max }=15$. A large part of the estimates are equal to 1 or 15 (values corresponding to the bounds). The reach length being around $8 \mathrm{~km}$, a time-delay equal to 1 means that an action on the upstream is propagated at a speed faster than $60 \mathrm{~m} / \mathrm{s}$, that is not realistic. Next, most of other estimates corresponds to the upper bound (30\%). In other terms, for $30 \%$ of the data sets, the time-delay is upper than 15 (and not that $\hat{d}=15$ ). So, the most frequent estimate is 1 which is not admissible. This approach gives unsuccessful outcomes. This failure can be explained by the fact that the model structure (8) does not match with the 'true' system (including the noise properties) [15]. Fig. 9b 


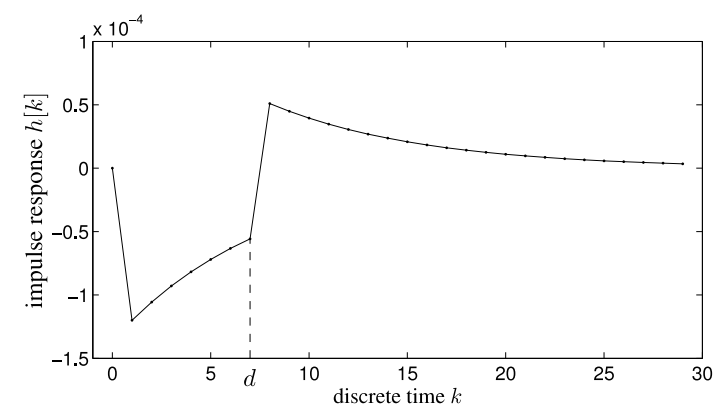

Fig. 10. Example of a theoretical impulse response $h[k]$ between the level $\Delta h_{L}$ (in $m$ ) and the inflow $\Delta u_{Q_{i}}\left(m^{3} / s\right)$.

represents the histogram of the time-delay estimates for the indirect approach. The results analysis leads to the same conclusion than the direct approach. This failure can be explain by the fact that the model structure (10) is based on the very simplified model of humain operators (9). In the continuation of this article, a FIR model between $\Delta u_{Q_{i}}$ and $\Delta h_{L}$ is considered. Its higher degree-of-freedom should allow to better take into account the operators behavior. The price to pay is a higher number of parameters to estimate and as a consequence a risk of ill-conditionned problem which can be resolved by introducing prior knowledge with a Bayesian approach.

The transfer function (10) can be used to get prior knowledge on the FIR model. It may be viewed as the sum of two first order models. The first one, with negative gain, is delayed of one sample, and the second one, with positive gain, is delayed of $d+1$ samples. Fig. 10 represents the theoretical impulse response between $\Delta h_{L}$ and $\Delta u_{Q_{i}}$ (with $d=7, K_{i} T_{s} / A=10^{-4} \mathrm{~s} / \mathrm{m}^{2}, K_{o} T_{s} / A=1.2 .10^{-4} \mathrm{~s} / \mathrm{m}^{2}, K_{Q}=1$ and $K_{h}=-1000$ ). This impulse response $h[k]$ has two "discontinuities": a first one between the points $h[0]$ and $h[1]$ and a second one between $h[d]$ and $h[d+1]$. Apart from these discontinuities, the impulse response varies slowly. It is thus possible to estimate the time-delay $d$ by detecting the second discontinuity.

From these observations, the following section presents the proposed Bayesian method for estimating jointly the FIR and the time-delay.

\section{JOINT IMPULSE RESPONSE AND TIME-DELAY ESTIMATION}

Consider a single-input single-output discrete-time linear dynamic model described by a system of linear equations based on the available data (let $N$ be the length of data set)

$$
\mathbf{y}=\mathbf{U h}+\mathbf{w}
$$

where $\mathbf{y}=[y[0], \cdots, y[N-1]]^{T} \in \mathbb{R}^{N}$ is the output vector, $\mathbf{h}=[h[0], \cdots, h[M-1]]^{T} \in \mathbb{R}^{M}$ is the unknown FIR, $M$ being its truncation order $(M<N), \mathbf{w}=[w[0], \cdots, w[N-1]]^{T} \in \mathbb{R}^{N}$ represents a noise term due to 
model, truncation errors and measurement uncertainties on data, and

$$
\mathbf{U}=\left[\begin{array}{cccc}
u[0] & 0 & \cdots & 0 \\
u[1] & u[0] & \ddots & \vdots \\
\vdots & \vdots & \ddots & \vdots \\
u[M-2] & u[M-3] & & 0 \\
u[M-1] & u[M-2] & & u[0] \\
\vdots & \vdots & & \vdots \\
u[N-1] & u[N-2] & \cdots & u[N-M]
\end{array}\right] \in \mathbb{R}^{N \times M}
$$

is a Tœplitz matrix composed of samples of the input $u$.

\section{A. Standard FIR estimator: the maximum likelihood}

If we assume that $\mathbf{w}$ is a zero-mean white Gaussian noise sequence with covariance matrix $\sigma_{w}^{2} \mathbf{I}$ and that $\mathbf{w}$ is independent of $\mathbf{h}$, an explicit expression of the maximum likelihood (ML) estimator is obtained

$$
\hat{\mathbf{h}}^{\mathrm{ML}}=\left(\mathbf{U}^{T} \mathbf{U}\right)^{-1} \mathbf{U}^{T} \mathbf{y}
$$

This is a well-posed problem (in sense of HADAMARD), but it can be ill-conditionned [19] if the condition number of $\mathbf{U}^{T} \mathbf{U}$ is high. In this case, the ML solution is unsuitable because it is too sensitive to data noise. A solution consists in taking into account other information on the FIR like the fact that it varies slowly.

\section{B. Bayesian FIR estimator: the maximum a posteriori}

The introduction of a priori information in the estimation problem is made by multiplying the likelihood function by the prior probability density function (pdf), which is representative of the available information. After normalization, we obtain the posterior pdf (Bayes' theorem)

$$
p_{\mathbf{H}}\left(\mathbf{h} \mid \mathbf{y}, \mathbf{U}, \boldsymbol{\beta}_{\mathbf{h}}, \boldsymbol{\beta}_{\mathbf{w}}\right)=\frac{p_{\mathbf{Y}}\left(\mathbf{y} \mid \mathbf{h}, \mathbf{U}, \boldsymbol{\beta}_{\mathbf{w}}\right) p_{\mathbf{H}}\left(\mathbf{h} \mid \boldsymbol{\beta}_{\mathbf{h}}\right)}{p_{\mathbf{Y}}\left(\mathbf{y} \mid \mathbf{U}, \boldsymbol{\beta}_{\mathbf{h}}, \boldsymbol{\beta}_{\mathbf{w}}\right)}
$$

where $p_{\mathbf{Y}}\left(\mathbf{y} \mid \mathbf{h}, \mathbf{U}, \boldsymbol{\beta}_{\mathbf{w}}\right)$ is the likelihood function, $p_{\mathbf{H}}\left(\mathbf{h} \mid \boldsymbol{\beta}_{\mathbf{h}}\right)$ is the prior pdf of $\mathbf{h}$ and $p_{\mathbf{Y}}\left(\mathbf{y} \mid \mathbf{U}, \boldsymbol{\beta}_{\mathbf{h}}, \boldsymbol{\beta}_{\mathbf{w}}\right)$, equal to the integral of the product of the likelihood function and the prior pdf, allows to normalize the product. The variables $\boldsymbol{\beta}_{\mathbf{h}}$ and $\boldsymbol{\beta}_{\mathbf{w}}$ represent the hyperparameters of the prior on $\mathbf{h}$ and $\mathbf{w}$, respectively. The maximum a posteriori (MAP) estimator provides the argument of the maximum of this posterior pdf.

The a priori information about the temporal smoothness of the FIR is introduced by considering the second derivatives $^{2}$ of $\mathbf{h}$ (second order finite differences approximations): $\mathbf{h}_{\mathbf{D}}=\mathbf{D h}$ where $\mathbf{D}$ is the following Tœplitz

\footnotetext{
${ }^{2}$ Superior derivatives could be used but for this application a penalization with second derivatives is sufficient.
} 
roughening matrix of dimension $M \times M$

$$
\mathbf{D}=\left[\begin{array}{cccc}
2 & -1 & & \\
-1 & \searrow & & 0 \\
& -1 & 2 & -1 \\
0 & & & -1
\end{array}\right]
$$

and by assuming that $\mathbf{h}_{\mathbf{D}}$ is a zero-mean white Gaussian noise sequence with covariance matrix $\sigma_{h_{\mathbf{D}}}^{2} \mathbf{I}$. The smoothness constraint is then parameterized by $\sigma_{h_{\mathbf{D}}}^{2}$ because a low value of $\sigma_{h_{\mathrm{D}}}^{2}$ means a low variation between the successive points of $\mathbf{h}$, and conversely. The vector $\mathbf{h}$ then follows a zero-mean Gaussian distribution with a covariance matrix $\sigma_{h_{\mathbf{D}}}^{2}\left(\mathbf{D}^{T} \mathbf{D}\right)^{-1}$. So the posterior pdf is proportional to

$$
\exp \left(-\frac{1}{2 \sigma_{w}^{2}}\|\mathbf{y}-\mathbf{U h}\|^{2}-\frac{1}{2 \sigma_{h_{\mathbf{D}}}^{2}}\|\mathbf{D h}\|^{2}\right)
$$

and its maximization (or the minimization of its negative logarithm) leads to the MAP estimator given by (under the inversion condition of the matrix $\left(\mathbf{U}^{T} \mathbf{U}+\alpha \mathbf{D}^{T} \mathbf{D}\right)$ )

$$
\hat{\mathbf{h}}^{\mathrm{MAP}}=\left(\mathbf{U}^{T} \mathbf{U}+\alpha \mathbf{D}^{T} \mathbf{D}\right)^{-1} \mathbf{U}^{T} \mathbf{y}
$$

where $\alpha=\sigma_{w}^{2} / \sigma_{h_{\mathrm{D}}}^{2}$. Nevertheless, this expression is obtained by assuming that the FIR is globally smooth. So, in our case, the smoothness constraint will reduce the amplitude of the discontinuity to be detected and thus will complicate the detection task. In order to avoid this problem, we propose a method introducing a smoothness constraint on the FIR while preserving the discontinuities.

\section{Bayesian FIR and time-delay estimator: joint maximum a posteriori}

In order to refine the a priori information coding, we propose to force a smoothness constraint on the FIR, except at times characterizing the two discontinuities between $h[0]$ and $h[1]$, and between $h[d]$ and $h[d+1]$, while estimating the time-delay. Note that the FIR estimate depends on the time-delay $d$.

The a priori information on the objects $\mathbf{h}$ and $d$ is expressed in terms of a joint prior pdf equal to: $p_{\mathbf{H}, D}\left(\mathbf{h}, d \mid \boldsymbol{\beta}_{\mathbf{h}}, \boldsymbol{\beta}_{d}\right)=$ $p_{\mathbf{H}}\left(\mathbf{h} \mid d, \boldsymbol{\beta}_{\mathbf{h}}\right) p_{D}\left(d \mid \boldsymbol{\beta}_{d}\right)$, where $\boldsymbol{\beta}_{\mathbf{h}}$ and $\boldsymbol{\beta}_{d}$ are vectors composed of the hyperparameters of the prior on $\mathbf{h}$ and $d$, respectively. The joint posterior pdf of $\mathbf{h}$ and $d, p_{\mathbf{H}, D}\left(\mathbf{h}, d \mid \mathbf{y}, \mathbf{U}, \boldsymbol{\beta}_{\mathbf{h}}, \boldsymbol{\beta}_{d}, \boldsymbol{\beta}_{\mathbf{w}}\right)$, combining the a priori information with the one of the data, is the following

$$
\frac{p_{\mathbf{Y}}\left(\mathbf{y} \mid \mathbf{h}, d, \mathbf{U}, \boldsymbol{\beta}_{\mathbf{w}}\right) p_{\mathbf{H}}\left(\mathbf{h} \mid d, \boldsymbol{\beta}_{\mathbf{h}}\right) p_{D}\left(d \mid \boldsymbol{\beta}_{d}\right)}{p_{\mathbf{Y}}\left(\mathbf{y} \mid \mathbf{U}, \boldsymbol{\beta}_{\mathbf{h}}, \boldsymbol{\beta}_{d}, \boldsymbol{\beta}_{\mathbf{w}}\right)} .
$$

We choose the estimator maximizing the joint posterior pdf, named joint maximum a posteriori (JMAP).

Always under the hypothesis that the noise is a zero-mean white Gaussian noise sequence with covariance matrix $\sigma_{w}^{2} \mathbf{I}$ and that it is independent of $\mathbf{h}$, the likelihood function is

$$
p_{\mathbf{Y}}\left(\mathbf{y} \mid \mathbf{h}, d, \mathbf{U}, \sigma_{w}^{2}\right)=\frac{1}{\left(2 \pi \sigma_{w}^{2}\right)^{N / 2}} \exp \left[-\frac{1}{2 \sigma_{w}^{2}}\|\mathbf{y}-\mathbf{U h}\|^{2}\right] .
$$




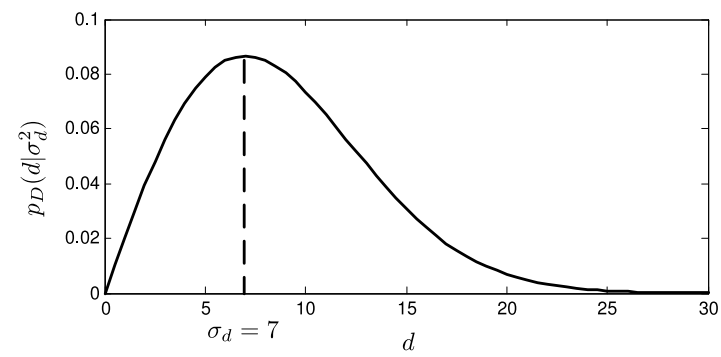

Fig. 11. The Rayleigh probability density function with $\sigma_{d}=7$.

We want to force the FIR to be relatively smooth, except between $h[0]$ and $h[1]$, and between $h[d]$ and $h[d+1]$. For that, we modify the matrix $\mathbf{D}$ in (19), used before to obtain a smoothness constraint on all the FIR. This new matrix, denoted by $\mathbf{D}_{d}$ (because it depends on $d$ ), is defined as follows

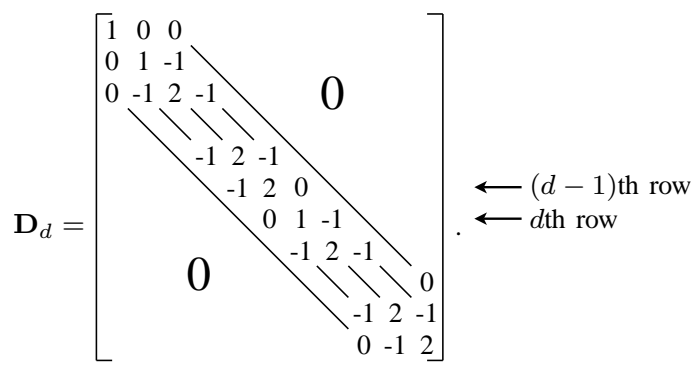

Note that the row $d-1$ contains $\left[\begin{array}{lll}-1 & 2 & 0\end{array}\right]$ instead of $\left[\begin{array}{lll}-1 & 1 & 0\end{array}\right]$. It is a small, but necessary, trick to obtain an invertible matrix. This change does not significantly modify the proposed approach. With this definition of $\mathbf{D}_{d}$, the vector $\mathbf{h}_{\mathbf{D}}=\mathbf{D}_{d} \mathbf{h}$ corresponds to the second derivatives of $\mathbf{h}$, except at times $0,1, d$ and $d+1$, so that no constraint is applied between $h[0]$ and $h[1]$, and between $h[d]$ and $h[d+1]$. Hence, the prior pdf is equal to

$$
p_{\mathbf{H}}\left(\mathbf{h} \mid d, \sigma_{h_{\mathbf{D}}}^{2}\right)=\frac{1}{\left(2 \pi \sigma_{h_{\mathbf{D}}}^{2}\right)^{M / 2}} \exp \left[-\frac{1}{2 \sigma_{h_{\mathbf{D}}}^{2}}\left\|\mathbf{D}_{d} \mathbf{h}\right\|^{2}\right]
$$

because $\operatorname{det}\left(\left(\mathbf{D}_{d}^{T} \mathbf{D}_{d}\right)^{-1}\right)=1$.

The a priori information about the time-delay is represented by a Rayleigh distribution whose expression is as follows

$$
p_{D}\left(d \mid \sigma_{d}^{2}\right)=\frac{d}{\sigma_{d}^{2}} \exp \left[-\frac{d^{2}}{2 \sigma_{d}^{2}}\right] \mathbb{I}_{[0,+\infty)}(d)
$$

where $\sigma_{d}=\arg \max _{d}\left[p_{D}\left(d \mid \sigma_{d}^{2}\right)\right]$ is a hyperparameter and $\mathbb{I}_{[0,+\infty)}(d)$ is the interval indicator function which is equal to 1 if $d \in[0,+\infty)$ and 0 otherwise. This law is depicted on Fig. 11 for $\sigma_{d}=7$. It has the advantages of taking into account the time-delay positivity ${ }^{3}$ and having a single hyperparameter whose value can be fixed proportionally to the reach length.

\footnotetext{
${ }^{3}$ In all rigor, the prior law on the delay should also be discrete in order to take into account the discrete character of the delay. However, this discrete character will only be considered at the optimization step.
} 
TABLE V

ESTIMATION ALGORITHM OF h AND $d$ WITH PREDETERMINED HYPERPARAMETERS.

$$
\begin{aligned}
& \diamond \text { For } d=d_{\min }, d_{\min }+1, \ldots, d_{\max }, \\
& \text { a) construction of the matrix } \mathbf{D}_{d}, \\
& \text { b) estimation of } \mathbf{h} \text { at } d \text { fixed: } \\
& \quad \widehat{\mathbf{h}}(d)=\left(\mathbf{U}^{T} \mathbf{U}+\alpha \mathbf{D}_{d}^{T} \mathbf{D}_{d}\right)^{-1} \mathbf{U}^{T} \mathbf{y} \\
& \text { c) computation of the criterion } J^{\mathrm{JMAP}}(\widehat{\mathbf{h}}(d), d) \\
& \diamond \hat{d}^{\mathrm{JMAP}}=\arg \underset{d \in \mathbb{D}\left(d_{\min }, d_{\max }\right)}{J^{\mathrm{JMAP}}(\widehat{\mathbf{h}}(d), d) .} \\
& \diamond \widehat{\mathbf{h}}^{\mathrm{JMAP}}=\widehat{\mathbf{h}}\left(\hat{d}^{\mathrm{JMAP}}\right)=\left(\mathbf{U}^{T} \mathbf{U}^{\mathrm{M}}+\alpha \mathbf{D}_{\hat{d}^{\mathrm{JMAP}}}^{T} \mathbf{D}_{\hat{d}^{\mathrm{JMAP}}}\right)^{-1} \mathbf{U}^{T} \mathbf{y} .
\end{aligned}
$$

The criterion to minimize $J^{\mathrm{JMAP}}(\mathbf{h}, d)$ is obtained by keeping the negative logarithm of the product of the terms of (23), (25) and (26) which depend of $\mathbf{h}$ and $d$. That gives $J^{\mathrm{JMAP}}(\mathbf{h}, d)$ equal to

$$
\underbrace{\|\mathbf{y}-\mathbf{U h}\|^{2}}_{\begin{array}{c}
\text { Fidelity to } \\
\text { data }
\end{array}}+\frac{\sigma_{w}^{2}}{\sigma_{h_{\mathbf{D}}}^{2}} \underbrace{\left\|\mathbf{D}_{d} \mathbf{h}\right\|^{2}}_{\begin{array}{c}
\text { Fidelity to } \\
\text { the } \text { a priori } \\
\text { on } \mathbf{h}
\end{array}}+\sigma_{\begin{array}{c}
\text { Fidelity to } \\
\text { the } \text { a priori on } d
\end{array}}^{\left(\frac{d^{2}}{\sigma_{d}^{2}}-2 \ln (d)\right)}
$$

for $\mathbf{h} \in \mathbb{R}^{M}$ and $d \in \mathbb{D}\left(d_{\min }, d_{\max }\right)$. The hyperparameter $\sigma_{h_{\mathbf{D}}}^{2}$ allows to adjust the a priori smoothness constraint on $\mathbf{h}$ and the noise variance $\sigma_{w}^{2}$ allows to adjust a compromise between the fidelity to data and the fidelity to the prior on $\mathbf{h}$ and $d$. Note that if we do not have prior on the time-delay, it is possible to consider a uniform distribution (instead of the Rayleigh pdf), which leads to the following criterion

$$
J_{\text {unif }}^{\mathrm{JMAP}}(\mathbf{h}, d)=\|\mathbf{y}-\mathbf{U h}\|^{2}+\frac{\sigma_{w}^{2}}{\sigma_{h_{\mathbf{D}}}^{2}}\left\|\mathbf{D}_{d} \mathbf{h}\right\|^{2} .
$$

The criterion $J^{\mathrm{JMAP}}(\mathbf{h}, d)$ is quadratic in $\mathbf{h}$ and, at a fixed $d$, has an explicit solution

$$
\widehat{\mathbf{h}}^{\mathrm{JMAP}}(d)=\left(\mathbf{U}^{T} \mathbf{U}+\alpha \mathbf{D}_{d}^{T} \mathbf{D}_{d}\right)^{-1} \mathbf{U}^{T} \mathbf{y}
$$

where $\alpha=\sigma_{w}^{2} / \sigma_{h_{\mathrm{D}}}^{2}$ corresponds to a regularization parameter, but it is not convex in $d$. Nevertheless, the set of admissible time-delays $\mathbb{D}\left(d_{\min }, d_{\max }\right)$ is finite. So, a simple method consists in performing an exhaustive search by computing the criterion $J^{\mathrm{JMAP}}(\mathbf{h}, d)$ for all values of $\mathbb{D}\left(d_{\min }, d_{\max }\right)$. Finally, the estimation of $\mathbf{h}$ and $d$ are summarized by the algorithm $\mathrm{V}$.

However, if it is possible to fixe the single parameter $\alpha=\sigma_{w}^{2} / \sigma_{h_{\mathrm{D}}}^{2}$ for all data sets during the JMAP estimation with a uniform distribution (28) (because this hyperparameter only influences the smoothness constraint on the FIR), the two hyperparameters $\sigma_{w}^{2}$ and $\sigma_{h_{\mathrm{D}}}^{2}$ must be estimated in the case of a Rayleigh prior on $d$ (27) in order to accurately adjust the smoothness constraint and the influence of the prior on $d$. In conclusion the problem resolution requires the determination of the hyperparameters $\sigma_{h_{\mathrm{D}}}^{2}$ and $\sigma_{w}^{2}$ which is detailed in the next section. 


\section{HYPERPARAMETER ESTIMATION}

Hyperparameters constitute a second level of description of the problem, essential to the resolution of the first level constituted by parameters themselves [24]. Note that the joint posterior pdf of parameters $\mathbf{h}, d$ and hyperparameters $\sigma_{h_{\mathbf{D}}}^{2}$ and $\sigma_{w}^{2}$, denoted by $p\left(\mathbf{h}, d, \sigma_{h_{\mathbf{D}}}^{2}, \sigma_{w}^{2} \mid \mathbf{y}, \mathbf{U}, \sigma_{d}^{2}\right)$ and equal to

$$
\frac{p\left(\mathbf{y} \mid \mathbf{h}, d, \mathbf{U}, \sigma_{w}^{2}\right) p\left(\mathbf{h} \mid d, \sigma_{h_{\mathbf{D}}}^{2}\right) p\left(d \mid \sigma_{d}^{2}\right) p\left(\sigma_{h_{\mathbf{D}}}^{2}\right) p\left(\sigma_{w}^{2}\right)}{p\left(\mathbf{y} \mid \mathbf{U}, \sigma_{d}^{2}\right)}
$$

summarizes all information in both inference levels. So, it is interesting to jointly maximize it with respect to both parameters and hyperparameters. Moreover, if the hyperparameters $\sigma_{h_{\mathrm{D}}}^{2}$ and $\sigma_{w}^{2}$ each follow a uniform a priori distribution (the choice of an other non-informative prior distribution is possible), it follows that $p\left(\mathbf{h}, d, \sigma_{h_{\mathbf{D}}}^{2}, \sigma_{w}^{2} \mid \mathbf{y}, \mathbf{U}, \sigma_{d}^{2}\right.$ ) is proportional to

$$
\frac{p\left(\mathbf{y} \mid \mathbf{h}, d, \mathbf{U}, \sigma_{w}^{2}\right) p\left(\mathbf{h} \mid d, \sigma_{h_{\mathbf{D}}}^{2}\right) p\left(d \mid \sigma_{d}^{2}\right)}{p\left(\mathbf{y} \mid \mathbf{U}, \sigma_{d}^{2}\right)} .
$$

The problem is that the limit of this pdf (with the choices made in the previous section) tends towards infinity, whereas the samples of $\mathbf{h}$ and the hyperparameter $\sigma_{h_{\mathbf{D}}}^{2}$ tend towards zero. Consequently, the JMAP estimator, which maximizes this pdf, theoretically leads to a null $\mathrm{FIR}^{4}$ whatever data is.

The degeneracy of the likelihood function in the estimation framework of a Gaussian mixture is a known problem [25]. A solution consists to penalize the likelihood function with a prior pdf on the noise variance of inverse gamma type. Thus singularities are removed and the problem is well-posed [26], [27]. Based on the same idea, a solution to our problem consists to assume that $\sigma_{h_{\mathbf{D}}}^{2}$ follows a prior pdf of inverse gamma type so $p\left(\mathbf{h}, d, \sigma_{h_{\mathbf{D}}}^{2}, \sigma_{w}^{2} \mid \mathbf{y}, \mathbf{U}, \boldsymbol{\Theta}\right)$ is proportional to

$$
\frac{p\left(\mathbf{y} \mid \mathbf{h}, d, \mathbf{U}, \sigma_{w}^{2}\right) p\left(\mathbf{h} \mid d, \sigma_{h_{\mathbf{D}}}^{2}\right) p\left(d \mid \sigma_{d}^{2}\right) p\left(\sigma_{h_{\mathbf{D}}}^{2} \mid \alpha_{h}, \beta_{h}\right)}{p\left(\mathbf{y} \mid \mathbf{U}, \sigma_{d}^{2}\right)}
$$

with $p\left(\sigma_{h_{\mathbf{D}}}^{2} \mid \alpha_{h}, \beta_{h}\right)=\frac{\beta_{h}^{\alpha_{h}}}{\Gamma\left(\alpha_{h}\right)} \frac{\exp \left[-\frac{\beta_{h}}{\sigma_{h_{\mathbf{D}}}^{2}}\right]}{\left(\sigma_{h_{\mathbf{D}}}^{2}\right)^{\alpha_{h}+1}} \mathbb{I}_{[0,+\infty)}\left(\sigma_{h_{\mathbf{D}}}^{2}\right)$, where $\Gamma(\cdot)$ is the Gamma function, $\boldsymbol{\Theta}=\left\{\sigma_{d}^{2}, \alpha_{h}, \beta_{h}\right\}$ and $\left(\alpha_{h}, \beta_{h}\right) \in \mathbb{R}^{2+*}$. As a consequence (for $\sigma_{h_{\mathbf{D}}}^{2} \geqslant 0$ and $\left.d \geqslant 0\right), p\left(\mathbf{h}, d, \sigma_{h_{\mathbf{D}}}^{2}, \sigma_{w}^{2} \mid \mathbf{y}, \mathbf{U}, \boldsymbol{\Theta}\right)$ is proportional to

$$
\frac{d}{\left(\sigma_{w}^{2}\right)^{N / 2}\left(\sigma_{h_{\mathbf{D}}}^{2}\right)^{M / 2+\alpha_{h}+1} \sigma_{d}^{2}} \exp \left[-\frac{1}{2 \sigma_{w}^{2}}\|\mathbf{y}-\mathbf{U h}\|^{2}-\frac{1}{2 \sigma_{h_{\mathbf{D}}}^{2}}\left(\left\|\mathbf{D}_{d} \mathbf{h}\right\|^{2}+2 \beta_{h}\right)-\frac{d^{2}}{2 \sigma_{d}^{2}}\right]
$$

and we can verify that there is no more degeneracy problem.

Now, it remains to determine $\alpha_{h}$ and $\beta_{h}$. From the prior pdf of $\mathbf{h}(25)$, we can deduce the likelihood function of $\sigma_{h_{\mathbf{D}}}^{2}$

$$
p\left(\hat{\mathbf{h}} \mid \hat{d}, \sigma_{h_{\mathbf{D}}}^{2}\right)=\frac{1}{\left(2 \pi \sigma_{h_{D}}^{2}\right)^{M / 2}} \exp \left[-\frac{\left\|\mathbf{D}_{\hat{d}} \hat{\mathbf{h}}\right\|^{2}}{2 \sigma_{h_{D}}^{2}}\right]
$$

\footnotetext{
${ }^{4}$ In practice, the estimate can be not null if the optimization algorithm leads to a local maximum. Nevertheless, in the vast majority of these cases, we note that the smoothness constraint on the FIR is too important, that means that $\sigma_{h_{\mathrm{D}}}^{2}$ is too small.
} 
The posterior pdf is then

$$
p\left(\sigma_{h_{\mathbf{D}}}^{2} \mid \hat{\mathbf{h}}, \hat{d}, \alpha_{h}, \beta_{h}\right) \propto \frac{1}{\left(\sigma_{h_{\mathbf{D}}}^{2}\right)^{M / 2+\alpha_{h}+1}} \exp \left[-\frac{\nu / 2+\beta_{h}}{\sigma_{h_{D}}^{2}}\right]
$$

where $\nu=\left\|\mathbf{D}_{\hat{d}} \hat{\mathbf{h}}\right\|^{2}$. It is an inverse gamma distribution of parameters $\alpha_{0}=M / 2+\alpha_{h}$ and $\beta_{0}=\nu / 2+\beta_{h}$. Consequently, the parameters $\alpha_{0}$ and $\beta_{0}$ can be obtained from an appropriate shape of the pdf $p\left(\sigma_{h_{\mathrm{D}}}^{2} \mid \nu\right)$. The problem is that $\nu$ is unknown. Close information can be used instead: $\nu^{\mathrm{ML}}=\left\|\mathbf{D}_{\hat{d}} \hat{\mathbf{h}}^{\mathrm{ML}}\right\|^{2}$. Then we show that the choice $\alpha_{0}=M$ et $\beta_{0}=\nu^{\mathrm{ML}}$ leads to a suitable shape of $p\left(\sigma_{h_{\mathrm{D}}}^{2} \mid \nu\right)$ [17]. That yields to $\alpha_{h}=M / 2$ and $\beta_{h}=\nu^{\mathrm{ML}} / 2$.

The criterion to be minimized $J_{\alpha_{h}, \beta_{h}}^{\mathrm{JMAP}}\left(\mathbf{h}, d, \sigma_{h_{\mathbf{D}}}^{2}, \sigma_{w}^{2}\right)$ is

$$
\frac{1}{\sigma_{w}^{2}}\|\mathbf{y}-\mathbf{U h}\|^{2}+\frac{1}{\sigma_{h_{\mathbf{D}}}^{2}}\left(\left\|\mathbf{D}_{d} \mathbf{h}\right\|^{2}+2 \beta_{h}\right)+N \ln \left(\sigma_{w}^{2}\right)+\left(M+2\left(\alpha_{h}+1\right)\right) \ln \left(\sigma_{h_{\mathbf{D}}}^{2}\right)+\frac{d^{2}}{\sigma_{d}^{2}}-2 \ln (d) .
$$

The optimization problem can be solved by an iterative algorithm maximizing successively the pdf (33) according to the parameters $\mathbf{h}, d$, and then according to the hyperparameters $\sigma_{w}^{2}$ and $\sigma_{d}^{2}$

$$
\begin{gathered}
\left({\widehat{\sigma_{h_{\mathrm{D}}^{2}}}}^{(i)},{\widehat{\sigma_{w}^{2}}}^{(i)}\right)=\arg \max _{\sigma_{h_{\mathrm{D}}^{2}}, \sigma_{w}^{2}} p\left(\widehat{\mathbf{h}}^{(i-1)}, \widehat{d}^{(i-1)}, \sigma_{h_{\mathrm{D}}}^{2}, \sigma_{w}^{2}\right) \\
\left(\widehat{\mathbf{h}}^{(i)},{\widehat{d^{\prime}}}^{(i)}\right)=\arg \max _{\mathbf{h}, d} p\left(\mathbf{h}, d,{\widehat{\sigma_{h_{\mathrm{D}}^{2}}^{2}}}^{(i)},{\widehat{\sigma_{w}^{2}}}^{(i)}\right) .
\end{gathered}
$$

For $\sigma_{h_{\mathrm{D}}}^{2}$ and $\sigma_{w}^{2}$ fixed, minimizing the criterion (35) amounts to minimizing $J^{\mathrm{JMAP}}(\mathbf{h}, d)$. For $\mathbf{h}$ and $d$ fixed, this yields

$$
\begin{gathered}
\widehat{\sigma_{h_{\mathbf{D}}}^{2}}\left(\mathbf{h}, d, \sigma_{w}^{2}\right)=\frac{\left\|\mathbf{D}_{d} \mathbf{h}\right\|^{2}+2 \beta_{h}}{M+2\left(\alpha_{h}+1\right)} \\
\widehat{\sigma_{w}^{2}}\left(\mathbf{h}, d, \sigma_{h_{\mathbf{D}}}^{2}\right)=\frac{\|\mathbf{y}-\mathbf{U h}\|^{2}}{N} .
\end{gathered}
$$

Finally, the iterative algorithm based on (36) is given in table VI in which the subroutine for the estimation of $\mathbf{h}$ and $d$ (algorithm of table $\mathrm{V}$ ) is integrated. The algorithm convergence can be proved by inspecting the convergences of $\sigma_{h_{\mathrm{D}}}^{2}$ and $\sigma_{w}^{2}$ with iterations. The risk of convergence to a local minimum is minimized by choosing correctly the initialization parameters from a priori knowledge.

\section{APPLiCATION RESUlT}

Fig. 12 represents the FIR estimates obtained with the ML, MAP and JMAP estimators $\left(\hat{h}^{\mathrm{ML}}, \hat{h}^{\mathrm{MAP}}\right.$, and for the JMAP with and without prior information on the hyperparameter $\sigma_{h_{\mathbf{D}}}: \hat{h}^{\mathrm{JMAP}}$ and $\hat{h}^{\mathrm{JMAP} d e g}$, respectively) from a one-day data set. The hyperparameter of the MAP estimator is fixed to $\alpha=10000$. This quite high value can be explained: since $\alpha=\sigma_{w}^{2} / \sigma_{h_{\mathbf{D}}}^{2}$, it is all the higher as $\sigma_{w}^{2}$ is high (much noise) and $\sigma_{h_{\mathbf{D}}}^{2}$ is small (strong smoothness constraint). Parameters of the JMAP algorithm are: $d_{\text {init }}=10, d_{\min }=1, d_{\max }=15$ and $\sigma_{d}=6.5$. The ML solution is very rough, which can make it difficult to detect the "real" discontinuity. Note that a typical condition number for $U^{T} U$ is around $2.10^{4}$ and it can rise to $1.10^{5}$. The MAP solution is smoother but, unfortunately, the discontinuity

\footnotetext{
${ }^{5}$ In the current state of this work, this choice is heuristic, obtained by successive tests.
} 
1. Initialization: $i=0, \widehat{\mathbf{h}}^{(i)}=\hat{\mathbf{h}}^{\mathrm{ML}}, \hat{d}^{(i)}=d_{\text {init }}$,

$\epsilon=10^{-3}, \alpha_{h}=M / 2, \beta_{h}=\left\|\mathbf{D} \hat{\mathbf{h}}^{\mathrm{ML}}\right\|^{2} / 2$.

2. Iteration: $i=i+1$.

$\diamond$ Estimation of $\sigma_{h_{\mathbf{D}}}^{2}:{\widehat{\sigma_{h_{\mathbf{D}}}^{2}}}^{(i)}=\frac{\left\|\mathbf{D}_{\hat{d}^{(i-1)}} \widehat{\mathbf{h}}^{(i-1)}\right\|^{2}+2 \beta_{h}}{M+2\left(\alpha_{h}+1\right)}$.

$\diamond$ Estimation of $\sigma_{w}^{2}:{\widehat{\sigma_{w}^{2}}}^{(i)}=\frac{\left\|\mathbf{y}-\mathbf{U h}^{(i-1)}\right\|^{2}}{N}$.

$\diamond$ For $d^{(i)}=d_{\min }, d_{\min }+1, \ldots, d_{\max }$,

a) construction of the matrix $\mathbf{D}_{d^{(i)}}$,

b) estimation of $\mathbf{h}^{(i)}$ with $d^{(i)}$ fixed:

$\widehat{\mathbf{h}}^{(i)}\left(d^{(i)}\right)=\left(\mathbf{U}^{T} \mathbf{U}+\frac{{\widehat{\sigma_{w}^{2}}}^{(i)}}{{\widehat{\sigma_{h_{\mathbf{D}}}^{2}}}^{(i)}} \mathbf{D}_{d^{(i)}}^{T} \mathbf{D}_{d^{(i)}}\right)^{-1} \mathbf{U}^{T} \mathbf{y}$,

c) computation of the criterion $J_{\alpha_{h}, \beta_{h}}^{\mathrm{JMAP}}\left(\widehat{\mathbf{h}}^{(i)}\left(d^{(i)}\right), d^{(i)}\right)$

$\diamond \hat{d}^{(i)}=\arg \min _{d^{(i)} \in \mathbb{D}\left(d_{\min }, d_{\max }\right)} J_{\alpha_{h}, \beta_{h}}^{\mathrm{JMAP}}\left(\widehat{\mathbf{h}}^{(i)}\left(d^{(i)}\right), d^{(i)}\right)$.

$\diamond \widehat{\mathbf{h}}^{(i)}=\left(\mathbf{U}^{T} \mathbf{U}+\frac{{\widehat{\sigma_{w}^{2}}}^{(i)}{\widehat{\sigma_{h_{\mathbf{D}}}^{2}}}^{(i)}}{\mathbf{D}_{\hat{d}^{(i)}}^{T}} \mathbf{D}_{\hat{d}^{(i)}}\right)^{-1} \mathbf{U}^{T} \mathbf{y}$.

3. Continue the iteration (step 2.) while

$$
\left|\frac{{\widehat{\sigma_{h_{\mathbf{D}}^{2}}}}^{(i)}-{\widehat{\sigma_{h_{\mathbf{D}}}^{2}}}^{(i-1)}}{{\widehat{\sigma_{h_{\mathbf{D}}}^{2}}}^{(i-1)}}\right|<\epsilon \text { and }\left|\frac{{\widehat{\sigma_{w}^{2}}}^{(i)}-{\widehat{\sigma_{w}^{2}}}^{(i-1)}}{{\widehat{\sigma_{w}^{2}}}^{(i-1)}}\right|<\epsilon .
$$

4. $\widehat{\mathbf{h}}^{\mathrm{MMAP}}=\widehat{\mathbf{h}}^{(i)}$ and $\hat{d}^{\mathrm{JMAP}}=\hat{d}^{(i)}$.

to detect is very attenuated. The estimate $\hat{h}^{\mathrm{JMAP} d e g}$ illustrates the degeneracy problem. The estimator has converged towards a local extremum: the solution is not null, but the smoothness constraint is too high. Moreover, the estimated time-delay is not very realistic: $\hat{d}^{\mathrm{JMAP} d e g}=1$. Finally, the JMAP solution is relatively smooth and preserves the discontinuity. The time-delay estimate is $\hat{d}^{\mathrm{JMAP}}=7$. Note that the same result is obtained with $\sigma_{d}=10$ for this data set.

We have at our disposal 332 data sets, relative to as many days, i.e. $N \approx 650$ with $T_{s}=133 s$ (see Fig. 7 for a data set example). The FIR estimated for each data set is represented in the form of a "bar" where each coefficient is represented by a gray level corresponding to its value (from white for the lowest value — with sign- to black for the highest one). For example, the $\hat{h}^{\text {JMAP }}$ bar is represented on the bottom of Fig. 12. This allows to observe the time evolution of the estimates by placing side by side all bars of the FIR estimated for each data set. This 


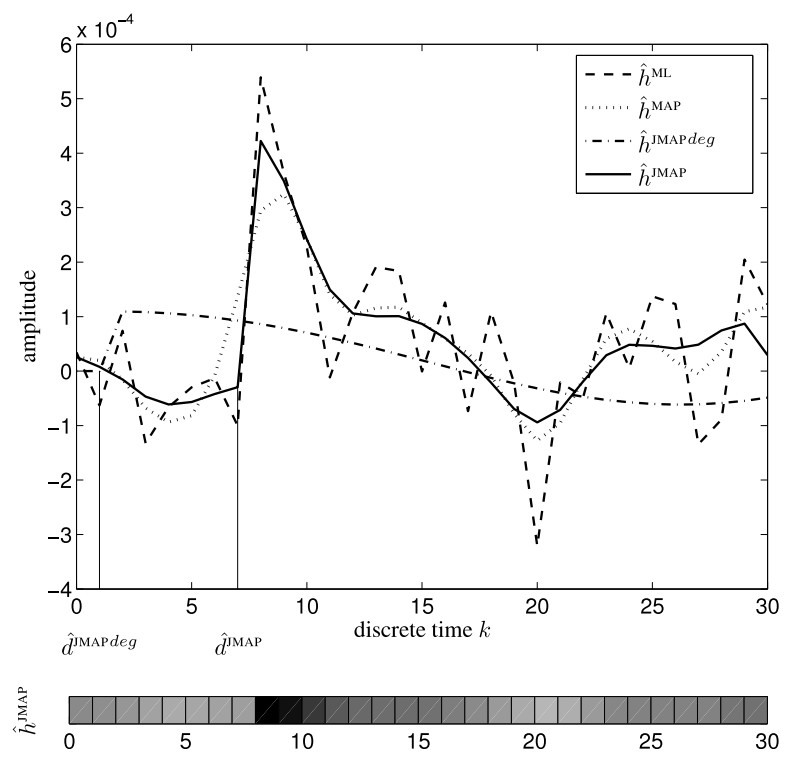

Fig. 12. Estimates of the impulse response and of the time-delay.

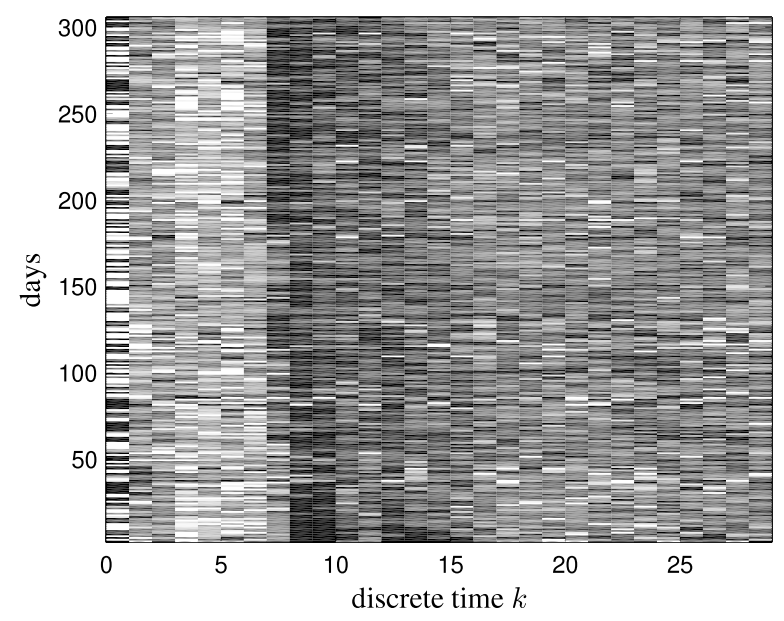

Fig. 13. TDR of the impulse responses estimated by the ML estimator.

juxtaposition creates a "time-day representation" (TDR) as presented in Fig. 13 to 16.

Fig. 13 represents the TDR of FIR estimated by the ML estimator over one year. The solutions are very rough but the presence of two discontinuities, characterized by a high gray level change, can be observed. Note that this estimator does not take into account their presence. This result corroborates the simplified model obtained in section III and especially consolidates the assumption (and the prior knowledge) of the existence of two discontinuities. A visual analysis indicates that the majority of the time-delays lies between 6 and 7 . It is possible to estimate the time-delays by detecting a change in the mean of these FIR estimates with for example the CUSUM algorithm [28], but in order to give satisfactory results this algorithm requires a parameter tuning for each data set 


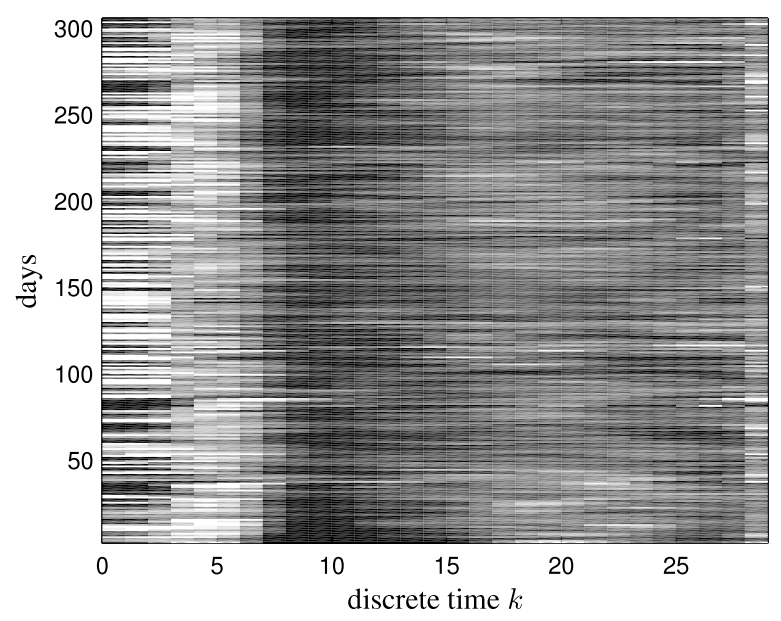

Fig. 14. TDR of the impulse responses estimated by the MAP estimator.

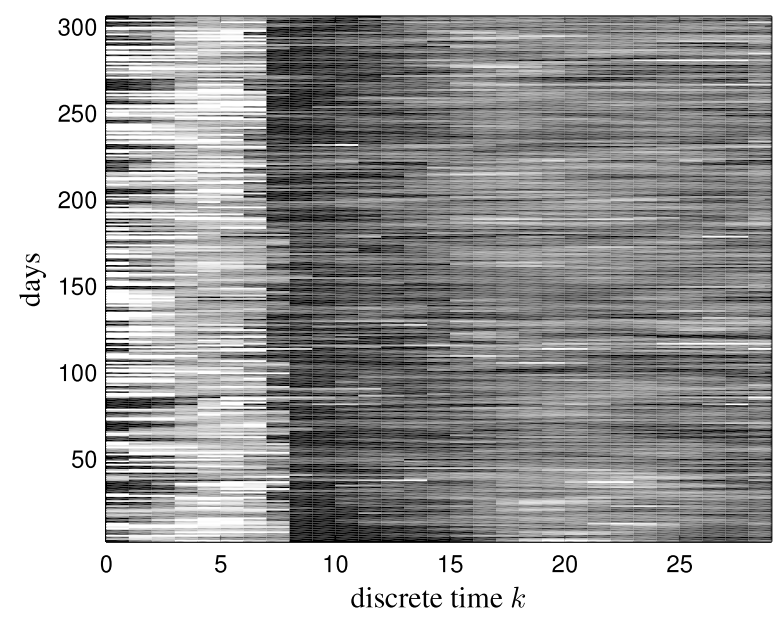

Fig. 15. TDR of the impulse responses estimated by the JMAP estimator (uniform pdf with $\alpha$ fixed to 10000).

(different estimation error), which is tedious with more than 300 data sets.

Fig. 14 represents the FIR estimates obtained with the MAP estimator with a regularization hyperparameter $\alpha=10000$. As expected, the estimates are smoother than those obtained with the ML estimator. This is reflected by weak variations of the gray levels between the samples (in $k$-coordinate direction). However, the discontinuities are less marked than previously, thus making the visual detection of the time-delay more tricky. Just like for the ML method, it is also possible to use the CUSUM algorithm to estimate the time-delay. Nevertheless, the time-delay estimated by the detector will be imprecise (detection delay) because of the smoothness constraint.

Fig. 15 represents the FIR estimates obtained with the JMAP estimator (29) with a uniform distribution as prior on the time-delay and $\alpha$ fixed to 1000 . As it can be seen, the proposed method gives a better map in the sense that the discontinuity is highlighted. Moreover, the method gives directly access to the time-delay estimates (without the 


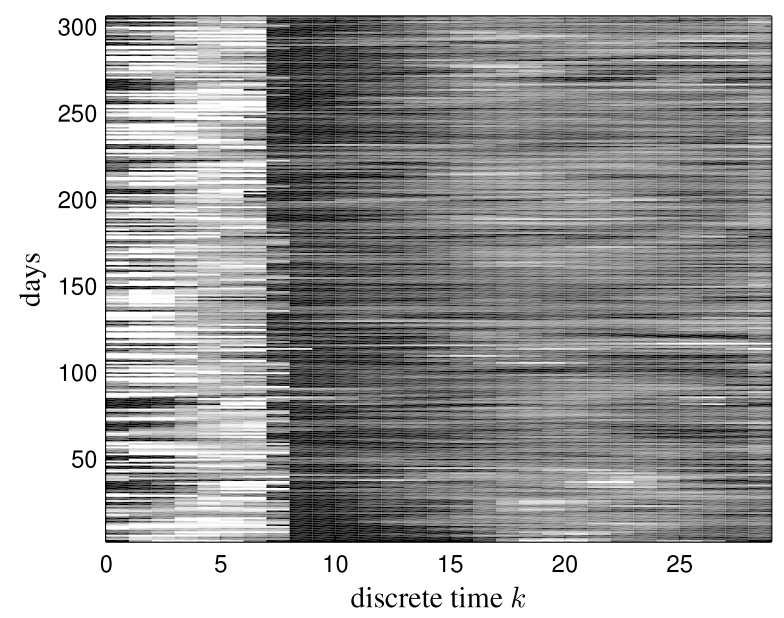

Fig. 16. TDR of the impulse responses estimated by the JMAP estimator (Rayleigh pdf).

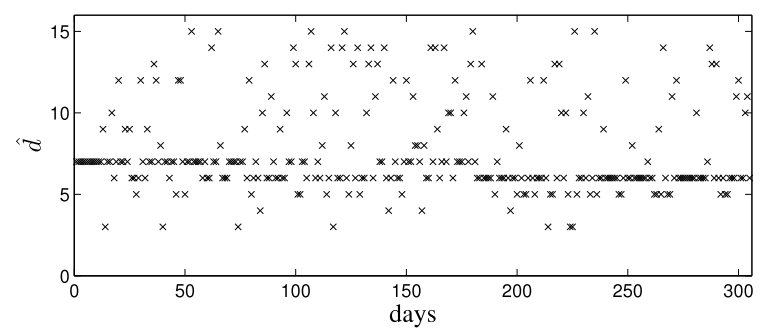

(a) with uniform prior on $d(\alpha=10000)$

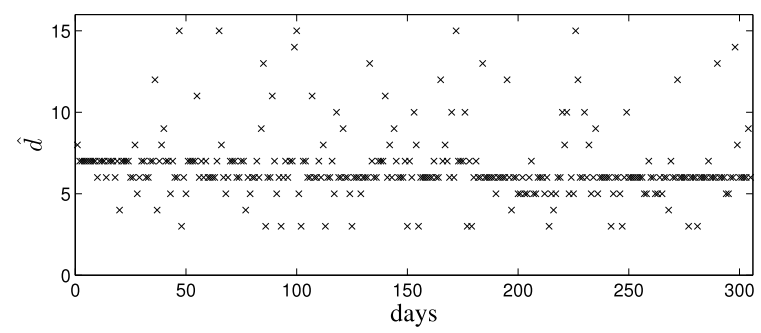

(b) with uniform prior on $d$ ( $\alpha$ estimated)

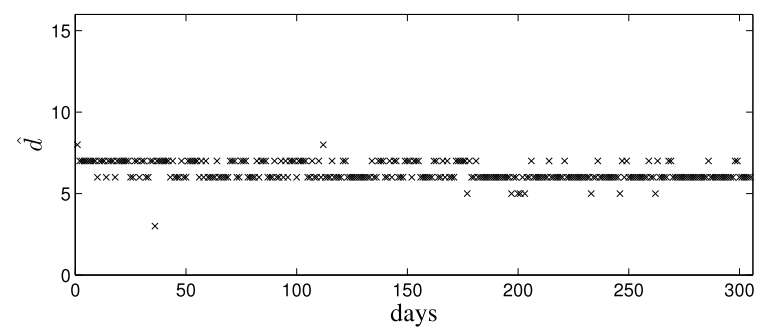

(c) with Rayleigh prior on $d$

Fig. 17. Time-delay estimates map (JMAP). 


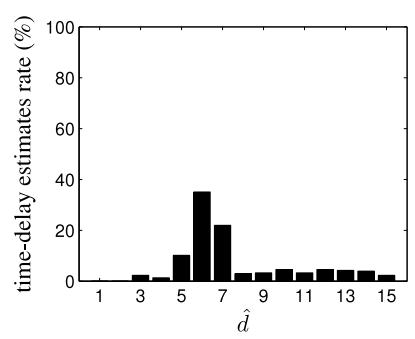

(a) with uniform prior on $d$ ( $\alpha=$ 10000)

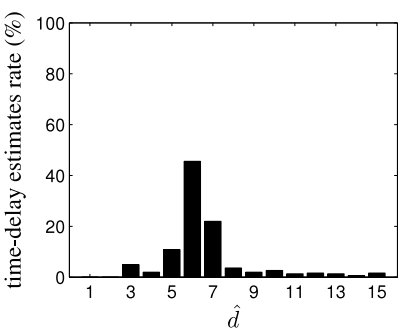

(b) with uniform prior on $d$ ( $\alpha$ estimated)

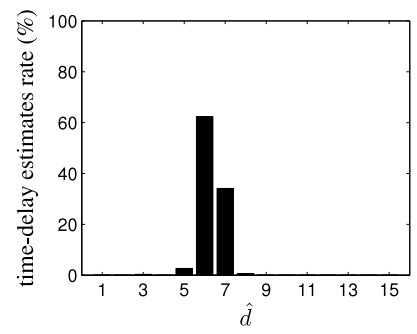

(c) with Rayleigh prior on $d$

Fig. 18. Histograms of the time-delay estimates (JMAP).

need of another detection algorithm). Fig. 17a and Fig. 18a represent the distribution over the year of the time-delay estimates and their histogram, respectively. A large part of the estimates lies between 5 and 7 with a uniform prior and this number increases when the hyperparameter $\alpha$ is estimated as shown Fig. 17b and Fig. 18b.

Fig. 16 represents the FIR estimates obtained with the JMAP estimator with a Rayleigh prior on $d$ by using the algorithm given in table VI. The distribution over the year of the time-delay estimates and their histogram are shown respectively on Fig. $18 \mathrm{~b}$ and 18c. More than $95 \%$ of the estimates are equal to 6 or 7 . This result tends to show that the time-delay variation over a year is weak. The reference value of this time-delay normally used by operators for the manual control of the reach corresponds to a $d$ between 6 and 12. As a result, we have sensibly reduced the uncertainty about the variation interval of the time-delay over a year. Of course, these results are based on a priori assumptions, and particularly on the time-delay one.

Nevertheless, by comparison with the propagation times evaluated from hydraulic analysis in section II, the estimated time-delay are closed to the propagation time of the gravity waves with a shift of one sampling time instant. This difference is explained in (8) where the time-delay between the inflow rate and the downstream water level is equal to $d+1$. Consequently, the estimation results show that the time-delay corresponds to the propagation time of the gravity waves (the fastest waves). Lastly, the fact that the time-delay is only weakly linked to the flow is justified by the hydraulic analysis of the section II.

The JMAP method (with uniform prior on $d$ ) has been applied on 4 river reaches, of different lengths, arranged in cascade along the "Basse-Isère" river (in France). The variation intervals of the time-delays estimated for each reach are shown on Fig. 19. This figure also represents the uncertainties intervals of the time-delays obtained from 


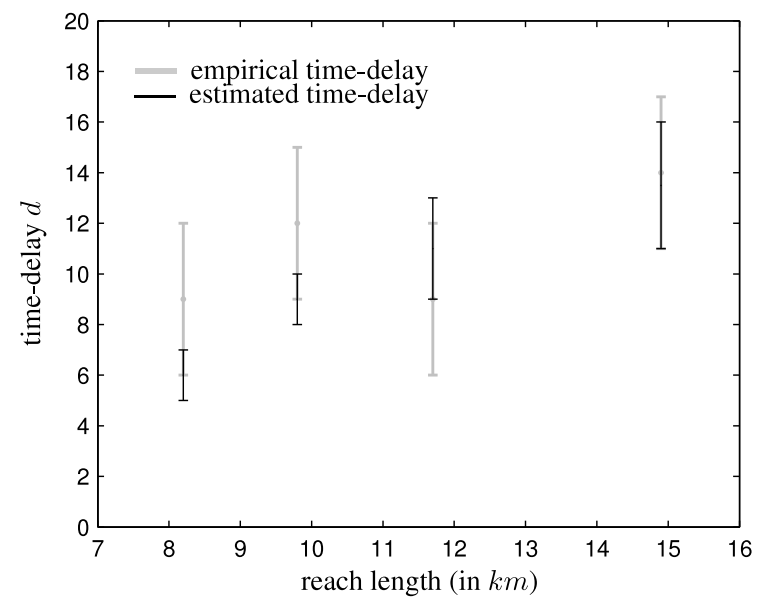

Fig. 19. Fluctuation intervals of empirical and estimated time-delays according to the reach length.

empirical knowledge. The latter are the reference values normally used by the operators for the manual control of the reaches. Note that the uncertainty of these estimates is approximately three sampling periods around the nominal value. Lastly, Fig. 19 also shows the significant improvement of the precision of the new estimates compared to the old ones since their variation intervals were at least divided by two for the first three reaches. The comparison of these results, with the ones of the section II, corroborates the relevance of the estimates and emphasizes the effectiveness of the proposed Bayesian identification approach in such an experimental context.

\section{CONCLUSION}

This paper focuses on the identification problem of a river reach managed by human operators from data sets which are daily collected in a production context. This practical issue is imposed by flood risks and fall-off in hydroelectric power production which prohibit any experimental protocol. The first difficulty lies in the model structure which contains a combined feedback/feedforward control carried out by a human operator. The second one comes from the few informative data sets due to the lack of persistent input signals. To compensate for these difficulties, a Bayesian method which jointly estimates the time-delay and a finite impulse response, is proposed. It is based on the fact that the time-delay introduces a discontinuity in the impulse response. The major difficulty encountered during this study is the degeneracy of the joint posterior probability density function during the hyperparameter estimation step. The suggested solution consists in penalizing this pdf by a prior pdf on the hyperparameter which is the cause of the degeneracy. The price of this change is the introduction of new parameters which are empirically tuned. This easy-to-implement method is applied to one-day data sets measured during one year. Results are described by a time-day representation which clearly points out a weak evolution of the estimated time-delay over a year. Lastly, these results are in accordance with the waves propagation times obtained from a hydraulic analysis of the reach. 


\section{REFERENCES}

[1] J. Schuurmans, O. H. Bosgra, and R. Brouwer, "Open-channel flow model approximation for controller design," Appl. Math. Modelling, vol. 19, pp. 525-530, 1995.

[2] B. Cuno and S. Theobald, "The relationship between control requirements, process complexity and modelling effort in the design process of river control systems," Math. Comput. Simul., vol. 46, pp. 611-619, 1998.

[3] X. Litrico, "Modeling, identification and robust control of open-channel hydraulic systems (in french)," Ph.D. dissertation, École Nationale du Génie Rural, des Eaux et Forêts, Montpellier, France, 1999.

[4] D. Georges and X. Litrico, Eds., Automatique pour la gestion des ressources en eau, ser. Systèmes automatisés, Traité IC2. Paris: Hermès Science Publications, 2002.

[5] X. Litrico, "Robust IMC flow control of SIMO dam-river open-channel systems," IEEE Trans. Control Syst. Technol., vol. 10, no. 3, pp. 432-437, 2002.

[6] X. Litrico and V. Fromion, "Analytical approximation of open-channel flow for controller design," Appl. Math. Modelling, vol. 28, no. 7, pp. 677-695, 2004.

[7] I. Mareels, E. Weyer, S. Ooi, M. Cantoni, Y. Li, and G. Nair, "Systems engineering for irrigation systems: Successes and challenges," Ann. Rev. Contr., vol. 29, pp. 191-204, 2005.

[8] X. Litrico and V. Fromion, " $H_{\infty}$ control of an irrigation canal pool with a mixed control politics," IEEE Trans. Control Syst. Technol., vol. 14, no. 1, pp. 99-111, 2006.

[9] E. Weyer, "System identification of an open water channel," Control Eng. Pract., vol. 9, no. 12, pp. 1289-1299, 2001.

[10] S. K. Ooi, M. P. M. Krutzen, and E. Weyer, "On physical and data driven modelling of irrigation channels," Control Eng. Pract., vol. 13, pp. 461-471, 2005.

[11] K. Eurén and E. Weyer, "System identification of open water channels with undershot and overshot gates," Control Eng. Pract., vol. 15, no. 7, pp. 813-824, 2007.

[12] M. Thomassin, T. Bastogne, A. Richard, and A. Libaux, "A bayesian approach for time-delay estimation of a managed river reach in imposed experimental conditions," in Proc. IFAC Workshop on Time-Delay Systems, Rocquencourt, France, Sep. 8-10, 2003.

[13] S. Niculescu, E. Verriest, J.-M. Dion, and L. Dugard, "Stability and robust stability of time-delay systems: a guided tour," in Stability and control of time-delay systems, L. Dugard and E. Verriest, Eds. Springer-Verlag, London, 1998, pp. 1-71.

[14] D. Dumur, A. Libaux, and P. Boucher, "Robust RST control for 'Basse-Isère' run-of-river cascaded hydro-electric plants," in Proc. of the European Control Conference, Porto, Portugal, 2001, pp. 1952-1957.

[15] L. Ljung, System identification: theory for the user, 2nd ed. Englewood Cliffs, NJ: Prentice Hall, 1999.

[16] M. Thomassin, T. Bastogne, A. Richard, and A. Libaux, "Time-delay estimation of a managed river reach from supervisory data," in Proc. IFAC Symp. on System Identification, Rotterdam, The Netherlands, Aug. 27-29, 2003.

[17] M. Thomassin, "Time-delay estimation under normal operating conditions. Application to river reach identification (in french)," Ph.D. dissertation, Université Henri Poincaré, Nancy 1, France, 2005.

[18] H. Kurz and W. Goedecke, "Digital parameter adaptive control of processes with unknown dead time," Automatica, vol. 17, pp. 245-252, 1981.

[19] J. Idier, Ed., Approche bayésienne pour les problèmes inverses, ser. Traitement du signal et de l'image (Traité IC2). Paris: Hermès Science Publication, 2001.

[20] V. M. Ponce and D. B. Simons, "Shallow wave propagation in open channel flow," ASCE Journal of the Hydraulics Division, vol. 103, no. HY12, pp. 1461-1476, 1977.

[21] V. T. Chow, Open Channel Hydraulics. New York: McGraw-Hill, 1959.

[22] M. H. Chaudhry, Open Channel Flow. Englewood Cliffs, NJ: Prentice Hall, 1993.

[23] S. K. Ooi and E. Weyer, "Closed loop identification of an irrigation channel," in Proc. IEEE Conf. Decision and Control, vol. 5, Orlando, FL, USA, 2001, pp. 4338-4343.

[24] G. Demoment and J. Idier, "Approche bayésienne pour la résolution des problèmes inverses en imagerie," in Problèmes inverses : de l'expérimentation à la modélisation, ser. Arago, M. Bonnet, Ed. Paris: Observatoire français des techniques avancées, 1999, vol. 22, pp. 59-77. 
[25] J. Kiefer and J. Wolfowitz, "Consistency of the maximum likelihood estimator in the presence of infinitely many incidental parameters," Ann. Math. Stat., vol. 27, no. 4, pp. 887-906, 1956.

[26] A. Ridolfi and J. Idier, "Penalized maximum likelihood estimation for univariate normal mixture distributions," in Proc. GRETSI Symp. on Signal and Image Processing, 1999, pp. 259-262.

[27] H. Snoussi and A. Mohammad-Djafari, "Penalized maximum likelihood for multivariate gaussian mixture," in Proc. AIP Conf., vol. 617, no. 1 , 2002, pp. 36-46.

[28] M. Basseville and I. M. Nikiforov, Detection of abrupt change. Theory and application. Englewood Cliffs, NJ: Prentice-Hall, 1993.

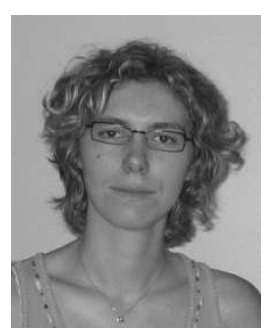

Magalie Thomassin was born in Vitry-le-François (France) in 1978. She received the Ph.D. degree in automatic control and signal processing from Nancy-Universite (France) at the Centre de Recherche en Automatique de Nancy (CRAN), in 2005. She is currently associate professor in the laboratoire de l'Intégration du Matériau au Système (IMS) at University of Bordeaux (France). Her research interests include system identification and statistical signal processing with application to open-channel flow systems and more recently to fractional systems.

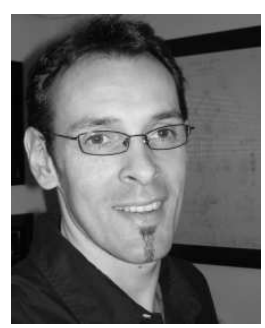

Thierry Bastogne was born in 1968 in Vitry-le-François, France. He received the M.Sc. degree in Computer Engineering and Optoelectronics from Nancy-Université, France in 1992. From 1992 to 1993 he was an engineer in an automation engineering company. He received the M.Sc. and Ph.D. degrees in Automatic Control Engineering from NancyUniversité in 1994 and 1997 respectively. Since 1998, he has been an assistant professor in the Automatic Control Department of Université Henri Poincaré (Faculté des Sciences et Techniques) and a member of the Centre de Recherche en Automatique de Nancy (CRAN). His current research interests deal with issues of system identification and their applications in systems biology and biomedical engineering.

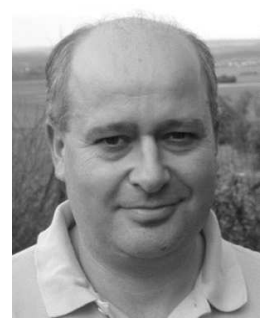

Alain Richard (M'93) received the Ph.D. degree from INPL Institut National Polytechnique de Lorraine, Nancy, France, in 1985 and the Habilitation diriger des recherches from Henri Poincaré University, Nancy, in 1993. From 1987 to 1993 he was with Henri Poincaré University as an Assistant Professor in electrical engineering. Since 1993, he has been Professor in the Department of Electrical Engineering at Henri Poincaré University. He is now Director of the Centre de Recherche en Automatique de Nancy which is a joint laboratory between CNRS and Nancy-University. His research interests are in system identification and statistical signal processing. He was associate editor of the Journal Européen des Systèmes Automatisés from 2003-2007, and a member of the IFAC Modelling, Identification and Signal Processing Technical Committee from 2000-2005. He is a member of IEEE Control Systems Society and IEEE Signal Processing Society. 